\title{
Functional Significance of Tie2 Signaling in the Adult Vasculature
}

\author{
Kevin G. Peters, ${ }^{*}$ Christopher D. Kontos, ${ }^{\dagger}$ P. Charles Lin, ${ }^{\ddagger}$ \\ Adrianne L. Wong, ${ }^{\|}$Prema Rao, ${ }^{\mathbb{q}}$ Liwen Huang, ${ }^{*} *$ Mark W. Dewhirst ${ }^{\dagger \dagger}$ \\ AND SABIta SANKaR \\ *Procter and Gamble Pharmaceuticals, Health Care Research Center, Mason, Ohio 45140; \\ ${ }^{\top}$ Department of Medicine, Division of Cardiology and Department of Pharmacology and Cancer \\ Biology, Duke University Medical Center, Durham, North Carolina 27710; ${ }^{*}$ Vanderbilt University \\ Medical Center, Nashville, Tennessee 37232-6300; "Genetics and Development, Columbia \\ University, New York, New York 10032; "Division of Urology, Department of Surgery, University \\ of Nebraska Medical Center, Omaha, Nebraska 68198-2360; **Myriad Pharmaceutics Inc., Salt \\ Lake City, Utah 84108; ${ }^{\dagger \dagger}$ Department of Radiation Oncology, Duke University Medical Center \\ Durham, North Carolina 27710; ${ }^{*}$ Celgene Corporation, San Diego, California 92121
}

\section{ABSTRACT}

Abundant data now demonstrate that the growth of new blood vessels, termed angiogenesis, plays both pathological and beneficial roles in human disease. Based on these data, a tremendous effort has been undertaken to understand the molecular mechanisms that drive blood vessel growth in adult tissues. Tie 2 recently was identified as a receptor tyrosine kinase expressed principally on vascular endothelium. Disrupting Tie2 function in mice resulted in embryonic lethality with defects in embryonic vasculature, suggesting a role in blood vessel maturation and maintenance. Based on these studies, we undertook a series of studies to probe the function of Tie 2 in adult vasculature that will form the focus of this chapter. Consistent with a role in blood vessel growth in adult vasculature, Tie2 was upregulated and activated in the endothelium of rat ovary and in healing rat skin wounds, both areas of active angiogenesis. Moreover, Tie2 was upregulated in the endothelium of vascular "hot spots" in human breast cancer specimens. Surprisingly, Tie2 also was expressed and activated in the endothelium of all normal rat tissues examined, suggesting a role in maintenance of adult vasculature. To determine the functional role of Tie2 in tumor vasculature, a soluble Tie 2 extracellular domain (ExTek) was designed that blocked the activation of Tie2 by its activating ligand, angiopoietin 1 (Ang1). Administration of recombinant ExTek protein or an ExTek adenovirus inhibited tumor growth and metastasis in rodent tumor models, demonstrating a functional role for Tie2 in pathological angiogenesis in adult tissues. To begin to understand the endothelial signaling pathways and cellular responses that mediate Tie 2 function, we identified signaling molecules that are recruited to the activated, autophosphorylated Tie2 kinase domain. Two of these molecules, SHP2 and GRB2, are part of the pathway upstream of mitogen-activated protein kinase (MAPK) activation, a pathway that may be responsible for morphogenetic effects of Tie 2 on endothelial cells. Another signaling molecule, p85, is responsible for recruitment of phosphatidylinositol 3 kinase (PI3-K) and activation of the Akt/PI3-K pathway. Akt/PI3-K has emerged as a critical pathway downstream of Tie2 that is necessary for cell survival effects as well as for chemotaxis, activation of endothelial nitric oxide synthase, and perhaps for anti-inflammatory effects of Tie2 activation. Taken together, 
these studies and many others demonstrate that the Tie2 pathway has important functions in adult tissues, in both quiescent vasculature and during angiogenesis, and help to validate the Tie2 pathway as a therapeutic target.

\section{Introduction}

Angiogenesis is the complex and dynamic process by which blood vessels are assembled and remodeled to form a functional vasculature. A large body of evidence has shown that angiogenesis is a crucial event for normal embryonic development. In adulthood, the vasculature is notably quiescent but angiogenesis is required for normal female reproductive function and likely is required for wound healing. Importantly, however, angiogenesis contributes to the pathogenesis of a number of diseases, including cancer, arthritis, obesity, atherosclerosis, and two common causes of blindness, diabetic retinopathy and macular degeneration (Folkman, 2001). Conversely, other diseases (e.g., coronary artery disease, peripheral vascular disease) are characterized by failure of the compensatory angiogenic response. Involvement of angiogenesis, or failure of angiogenesis, in these important diseases has led to a tremendous effort on the part of academic and industry researchers to define the molecular mechanisms that drive blood vessel assembly and remodeling.

Of the molecular mechanisms identified to date, activation of endothelial receptor tyrosine kinases (RTKs) by polypeptide growth factors appears to play a pivotal role in blood vessel growth and differentiation (Yancopoulos et al., 2000; Conway et al., 2001). Indeed, growth factors such as fibroblast growth factor, hepatocyte growth factor, platelet-derived growth factor, and the ephrins have been shown to modulate angiogenesis, suggesting that signaling by multiple RTKs is required for the proper assembly of blood vessels. Despite the role of these factors in angiogenesis, the expression of their cognate RTKs outside of the vasculature has dampened enthusiasm for these pathways as therapeutic targets. Importantly, RTKs for two families of angiogenic growth factors - the vascular endothelial growth factor family (VEGF) and the angiopoietin (Ang) family — are expressed predominantly on vascular endothelial cells, making them attractive targets for both pro- and anti-angiogenic therapy (Yancopoulos et al., 2000; Conway et al., 2001; Jones et al., 2001a; Loughna and Sato, 2001). This review will focus on work from our laboratory to elucidate the role of the Ang RTK, Tie2, in adult vasculature. The elegant and important work from our colleagues is cited where it has provided context for our studies and where it has confirmed or extended our work.

\section{Function of Ang/Tie2 Signaling in the Embryonic Vasculature}

Tie2 originally was described as the second member of an orphan RTK subfamily expressed predominantly in the embryonic endothelium (Dumont $e t$ 
al., 1992; Iwama et al., 1993; Maisonpierre et al., 1993; Runting et al., 1993; Sato et al., 1993; Schnurch and Risau, 1993; Ziegler et al., 1993). Tie1, the original member of the subfamily, and Tie 2 share an identical domain structure, with an unusual N-terminal ligand-binding domain, a single transmembrane domain, and an intracellular tyrosine kinase domain (Figure 1). Tie2 was found to be highly conserved across vertebrate species, predicting the importance of its biological function. In fact, the domain structure of Tie 2 is highly conserved from zebrafish to human, with the greatest amino acid homology occurring in the kinase domain (Figure 2) (Lyons et al., 1998).

Consistent with its expression pattern, disrupting the function of Tie 2 in transgenic mice resulted in early embryonic lethality secondary to vascular abnormalities (Dumont et al., 1994; Sato et al., 1995). Grossly, the patterning of large vessels such as the dorsal aorta was normal. However, Tie2-/- embryos failed to develop the normal hierarchy of vascular elements, suggestive of a failure of vascular branching and differentiation. Ultrastructurally, vessels of

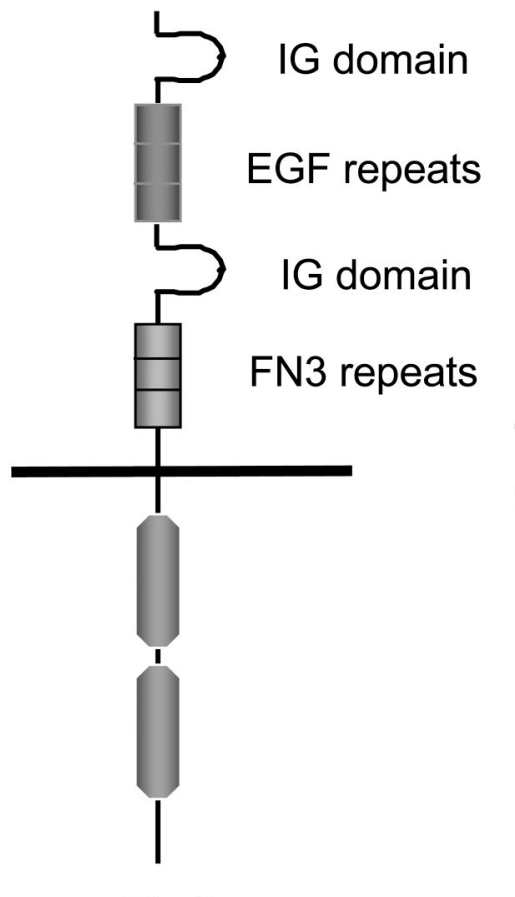

\section{Transmembrane domain}

\section{Kinase \\ domain}

\section{Tie2}

FIG. 1. Tie2 is a receptor tyrosine kinase with a unique extracellular ligand-binding domain comprised of immunoglobulin (IG) domains, epithelial growth factor (EGF) repeats, and fibronectinlike 3 (FN3) repeats. 


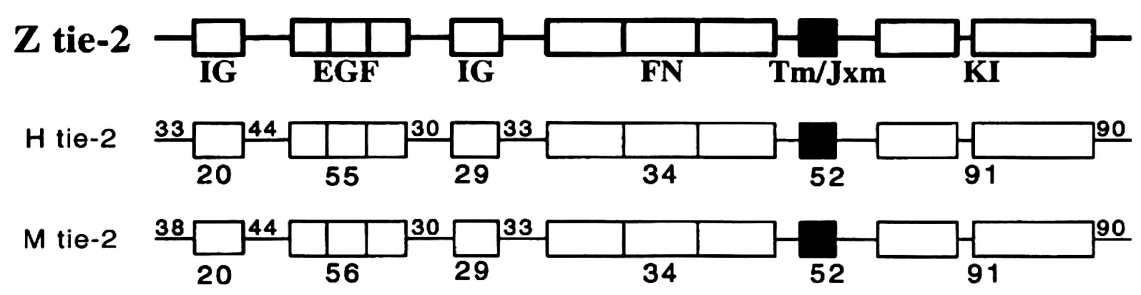

FIG. 2. Tie2 is highly conserved among vertebrate species from zebrafish to human, with the highest homology in the kinase domain.

Tie2-/- embryos showed a decreased number of endothelial cells and decreased contact between endothelial cells and the underlying perivascular cells (pericytes and smooth muscle cells), suggesting a role in the maturation and stabilization of the embryonic vasculature.

Tie2 was deorphanized with the discovery of the angiopoietins, Ang1 and Ang 2 (Davis et al., 1996; Maisonpierre et al., 1997). As is the case with other RTKs, Ang1 binding stimulated autophosphorylation of the kinase domain of Tie2. However, unlike activation of most growth factor RTKs, Ang1 activation of Tie2 did not stimulate mitogenesis, suggesting a novel role in endothelial biology. In contrast to Ang1, Ang2 did not stimulate Tie2 autophosphorylation but instead blocked Ang1-mediated Tie2 activation and endothelial migration, suggesting that Ang2 was a naturally occurring inhibitor of Tie2 activation (Maisonpierre et al., 1997; Witzenbichler et al., 1998; Teichert-Kuliszewska et al., 2001). Consistent with this finding, mice lacking functional Ang1 expression and mice overexpressing Ang2 both displayed a phenotype similar to Tie2-/mice (Suri et al., 1996; Maisonpierre et al., 1997). Recently, however, it has been demonstrated that, under some circumstances, Ang2 can stimulate Tie2, suggesting that the action of Ang2 as a Tie2 agonist or antagonist is context dependent (Kim et al., 2000b; Teichert-Kuliszewska et al., 2001; Gale et al., 2002). Two other Tie2 ligands have been identified that have disparate effects on Tie2 activation (Valenzuela et al., 1999). Demonstrating their selectivity, none of the Tie2 ligands identified to date bind to or activate the closely related Tie1 RTK. Taken together, these findings indicate that the Ang/Tie 2 pathway is a finely regulated one that plays crucial roles in the remodeling and maturation/stabilization of the embryonic vasculature. Based on these studies, we hypothesized that Tie2 signaling would have important roles in the normally quiescent adult vasculature.

\section{Role of Tie2 in the Normal Adult Vasculature}

To begin to explore the role of Tie 2 signaling in adult vasculature, it was first necessary to determine the pattern of Tie2 protein expression (Wong et al., 
1997). As anticipated from studies in mouse embryos, immunohistochemical analysis demonstrated that Tie2 was expressed in adult rat tissues undergoing angiogenesis. For example, during ovarian folliculogenesis, Tie 2 was expressed in the neovessels of the developing corpus luteum. Interestingly, Maisonpierre and colleagues found that Ang1 and Ang2 also were expressed in the corpus luteum, with Ang2 localizing to the leading edge of proliferating vessels and Ang1 localizing diffusely behind the leading edge (Maisonpierre et al., 1997). Based on this pattern of expression, it was suggested that Ang2-mediated inhibition of Tie2 activation serves to "destabilize" the vessel, to make it responsive to other angiogenic growth factors such as VEGF. Subsequently, Ang1-mediated activation of Tie2 would trigger remodeling and stabilization of the neovasculature.

Similar to the ovary, Tie2 was expressed on the endothelium of neovessels in skin wounds. By western analysis and RNase protection assay, Tie2 was expressed in normal skin, upregulated during active wound angiogenesis, and downregulated as newly formed vessels regressed. As an indicator of Tie2 activation, Tie2 phosphorylation could be detected in skin wounds at all stages of the healing process. Somewhat surprisingly, however, Tie2 also was expressed and phosphorylated in quiescent vasculature of normal skin. Taken together, the expression pattern in the ovary and in healing skin wounds suggested that, as in embryonic vasculature, the Tie2 pathway played a role in the development and remodeling of neovessels in adult vasculature.

The expression and apparent activation of Tie2 in the normally quiescent skin vasculature prompted a broader survey of Tie 2 in normal adult tissues. By immunohistochemistry, Tie2 was found to be expressed in endothelium of all adult tissues examined and in most, if not all, blood vessels, including arteries, veins, and capillaries. Studies in transgenic mice expressing marker transgenes driven by the Tie2 promoter have confirmed the broad expression of Tie 2 in adult vasculature (Schlaeger et al., 1997; Motoike et al., 2000). In fact, Tie2-cre transgenic mice now serve as a powerful tool to examine the role of more-broadly expressed genes, specifically in endothelium (Kisanuki et al., 2001; Theis et al., 2001; Forde et al., 2002; Takahashi et al., 2003; Vicent et al., 2003). In addition to its broad endothelial expression pattern, Tie2 phosphorylation was detected in every normal adult tissue examined, strongly suggesting a role for Tie 2 in the maintenance of quiescent adult vasculature.

Indeed, more-recent studies in transgenic and knockout (KO) mice suggest a critical role for Tie 2 in later stages of embryonic vascular development and in adult vasculature. Conditional expression of Tie2 in endothelium of mice homozygous for a Tie 2 null allele partially rescued the embryonic lethality of the Tie2 null phenotype (Jones et al., 2001b). Unfortunately, embryos were viable only until embryonic day (E) 15.5 and demonstrated signs of necrosis and vascular hemorrhage, suggesting that this approach did not allow a full rescue of 
the Tie 2 null phenotype. However, turning off Tie 2 expression with doxycycline in "rescued" animals dramatically increased the number of apoptotic endothelial cells, compared to doxycycline-treated, wild-type littermates. In support of these findings, mice chimeric for wild-type and doubly homozygous Tie2 and Tie1 null alleles lack mutant endothelial cells in the vasculature from E15.5 into adulthood (Puri et al., 1999). In contrast, endothelial cells doubly heterozygous for Tie 2 and Tie1 null alleles could be found in the embryonic vasculature but were completely absent in the adult vasculature. These findings demonstrate the importance of Tie 2 signaling in the later stages of embryonic development and strongly suggest a role for Tie 2 in the adult vasculature. However, further studies using conditional null alleles or perhaps more-effective "rescue" strategies are required to fully assess the role of Tie2 in quiescent adult vasculature.

\section{Role of Tie2 in Pathological Angiogenesis}

The expression and activation of Tie 2 during angiogenesis in normal adult vasculature suggested a role in pathological angiogenesis. To begin to understand the role of Tie2 in pathological neovascularization, Tie2 expression was assessed in a large number of human breast cancer tumor specimens (Peters et al., 1998). Consistent with results in adult rat vasculature, Tie2 was expressed in the vascular endothelium in both normal breast tissue and in breast tumors. However, the proportion of Tie2-positive tumor microvessels was increased in tumors, compared to normal breast tissue. Moreover, Tie2 expression was concentrated in "vascular hot spots" at the leading edge of invasive tumors. Subsequently, expression of Tie2 in human breast tumors has been confirmed and Tie2 expression has been shown in a number of other human tumors, including ovarian tumors, hepatocellular tumors, and glioblastoma (Stratmann et al., 1998; Hayes et al., 2000; Hata et al., 2002; Tanaka et al., 2002).

The expression of Tie 2 in tumor vasculature suggested a role for Tie 2 in tumor angiogenesis and in other settings where pathological angiogenesis contributes to disease progression. However, elucidating the functional significance of Tie2 expression in pathological angiogenesis required development of a specific inhibitor of Tie2 activity (Lin et al., 1997,1998a). Since angiopoietins bind with high affinity to the Tie2 extracellular domain, we reasoned that a soluble, recombinant Tie2 extracellular domain should be an efficient inhibitor of angiopoietin-mediated Tie 2 activity (Figure 3A). To explore this idea further, a fusion protein consisting of the full-length Tie2 extracellular domain with a 6-His tag (ExTek.6His) was constructed in a baculovirus expression vector and produced in insect cells. Purified ExTek.6His inhibited Ang1 and Ang2 binding to immobilized Tie2 very potently, with $\mathrm{IC}_{50} \mathrm{~s}$ of $110 \mathrm{nM}$ and $159 \mathrm{nM}$, respectively (Figure 3B). ExTek.6His also inhibited Ang1-mediated Tie2 phosphorylation and cell survival but had no effect on proliferation of a rat mammary tumor cell 


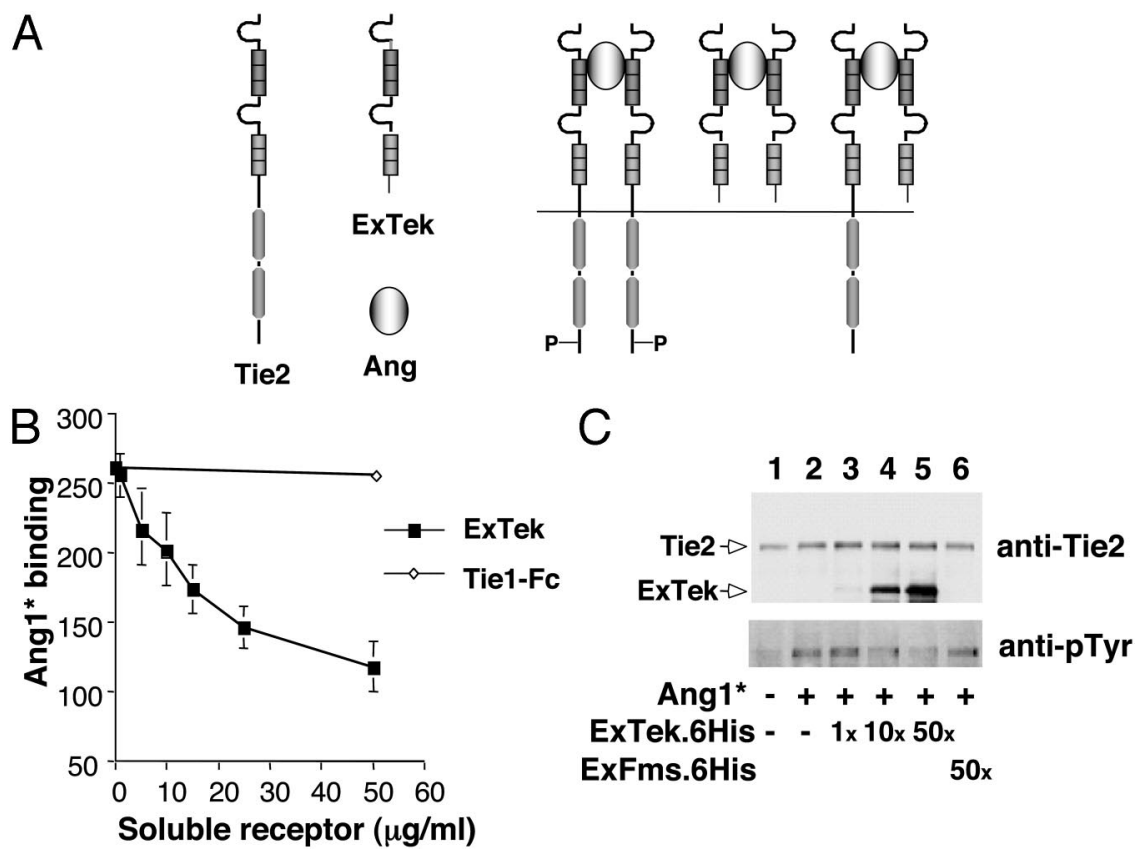

FIG. 3. ExTek is a potent inhibitor of Tie2 activation. (A) ExTek has two potential mechanisms of action. ExTek could simply sequester angiopoietin (Ang) and prevent binding to Tie2. Alternatively, ExTek could have a dominant-negative effect by binding both Ang and Tie2, to prevent proper oligomerization and activation of Tie2. (B) ExTek inhibited Ang $1 *$ binding to immobilized Tie2 extracellular domain. Binding of Ang1* (800 ng/ml) to immobilized Tie2-Fc was done in the presence of increasing ExTek concentrations. The Tie1-Fc was used as a control to demonstrate the specificity of the binding inhibition. (C) ExTek inhibited Ang1-stimulated Tie2 autophosphorylation. Serum-starved EAhy926 cells were stimulated with Ang $1^{*}(150 \mathrm{ng} / \mathrm{ml})$ plus the indicated amount of ExTek protein or control protein, ExFms (extracellular domain of c-fms). Tie2 and Tie2 phosphorylation levels then were detected by Tie2 immunoprecipitation and western blotting with anti-Tie2 and antiphosphotyrosine antibodies, respectively.

line. These results suggested that, as is the case for other RTKs, ligand-mediated dimerization was required for activation of Tie2 and that ExTek should be an effective inhibitor of Tie2 activation in vivo.

As an initial attempt to explore the role of Tie 2 in tumor angiogenesis, a single dose of recombinant ExTek was applied directly to rat mammary tumors grown in a transparent cutaneous window chamber (Lin et al., 1997). In this setting, ExTek-treated tumors grew more slowly (i.e., more than $75 \%$ reduction in tumor volume) and were less vascular (i.e., $40 \%$ reduction in tumor vessel length density) than tumors treated with a control protein. Confirming these results, systemic administration of ExTek using an adenoviral vector inhibited 
the growth and metastasis of two different murine tumors, a mammary carcinoma and a melanoma (Lin et al., 1998b). In particular, ExTek adenovirus markedly reduced the number and size of lung metastases when co-injected intravenously with tumor cells or when administered in a model of spontaneous metastatic tumor development. Interestingly, corneal angiogenesis induced by tumor cellconditioned medium was inhibited by recombinant ExTek, despite the presence of VEGF. Moreover, mammary tumor growth in the tumor window model was significantly inhibited by either recombinant ExTek or a recombinant VEGFR2 extracellular domain (Lin et al., 1997,1998a). These results suggest that Ang/ Tie 2 and VEGF pathways are both pivotal ones that are either independent or interdependent for tumor angiogenesis. Other studies independently employing different versions of the Tie2 extracellular domain have shown similar effects in different tumor models (Siemeister et al., 1999; Stratmann et al., 2001; Tanaka et al., 2002). Taken together, these findings indicate that Tie 2 signaling plays an important role in the development of the tumor vasculature and suggest that this pathway may be involved in the pathogenesis of other angiogenic diseases.

In light of these studies, it is surprising that Ang1 is not upregulated in most tumors. In fact, overexpression of Ang1 in tumor models has resulted in decreased tumor growth, an effect possibly related to Ang1-mediated stabilization of the tumor vasculature, making it resistant to angiogenic stimuli (Hayes et al., 2000; Shim et al., 2001,2002; Hawighorst et al., 2002; Stoeltzing et al., 2002,2003). These findings suggest that the inhibition of tumor angiogenesis by ExTek may not be due to a blockade of Ang1-mediated Tie2 activation. Rather, a number of studies have shown that Ang2 is upregulated in the tumor vasculature (Stratmann et al., 1998; Tanaka et al., 1999; Koga et al., 2001). Consistent with an active role in tumor angiogenesis, Ang2 is localized in the endothelium of proliferating neovessels at the interface between tumor and normal tissue (Holash et al., 1999; Zagzag et al., 1999). Based on these findings, it is possible that ExTek might function to inhibit some positive angiogenic function of Ang2 in tumor vasculature.

Consistent with this notion, recent studies have demonstrated that, in cultured endothelial cells, with longer exposures times or at higher concentrations, Ang2 can act as a Tie2 agonist (or perhaps a partial agonist) (Kim et al., 2000a; Teichert-Kuliszewska et al., 2001). In addition, mice carrying a homozygous Ang2 null allele have profound defects in the growth and patterning of lymphatic vasculature and a failure of remodeling and regression of the hyaloid vasculature that surrounds the neonatal lens (Gale et al., 2002). When the Ang2 gene was replaced with Ang1, the lymphatic defects, but not the vascular remodeling defects, were rescued, suggesting that Ang2 functions as a Tie2 antagonist in blood vasculature but as a Tie2 agonist in developing lymph vasculature. These findings suggest that the context dependence of Ang2 may be vascular bed specific. Whereas Ang2 normally is expressed in arterial smooth 
muscle, it appears to be expressed in endothelium only under conditions of vascular remodeling. Arterial expression of Ang2 suggests an as-yet-unknown role in arterial endothelial maintenance that may be related to its agonist effects during lymphatic remodeling. Interestingly, VEGFR3, a receptor that normally is expressed predominantly in adult lymphatic vasculature, was found to be upregulated in vascular endothelium of breast tumors (Valtola et al., 1999). It is tempting to speculate that at least some tumor vessels may take on a morelymphatic phenotype and thus respond to Ang2 as an agonist instead of an antagonist. Clearly, further work will be required to fully understand the biology of the Ang/Tie2 pathway in tumor vasculature.

The efficacy of ExTek in tumor models suggested a role for Tie2 in other angiogenic diseases. Similar to the tumor vasculature, Tie2 and Ang2 are upregulated in human retina in eyes from patients with ischemic retinal disorders (Takagi et al., 2003). In a mouse model of ischemic retinopathy, Ang2 expression was markedly upregulated in the ischemic retina and closely associated with retinal neovasculature (Hackett et al., 2000). In this setting, either local delivery of a recombinant, soluble Tie2-Fc by intravitreal injection or systemic delivery of ExTek by intramuscular injection of AdExTek inhibited retinal neovascularization (Hangai et al., 2001; Takagi et al., 2003). Similar results were obtained with AdExTek in a murine model of laser-induced choroidal neovascularization (Hangai et al., 2001). These findings, together with results using tumor models, establish a role for the Tie2 pathway in pathological angiogenesis and suggest that modulation of the Tie2 pathway could have broad application in the myriad of angiogenesis-dependent diseases.

\section{Tie2 Activation and Signal Transduction}

Clarification of the role of Tie2 in normal vasculature and in pathological angiogenesis will require a deeper understanding of the molecular and cellular mechanisms of Tie 2 activation and signal transduction. It is now generally accepted that RTK activation and signaling are initiated by ligand-mediated receptor dimerization (Schlessinger, 2000). Receptor dimerization results in cross-phosphorylation of each member of the dimer pair on specific tyrosine residues. Receptor cross-phosphorylation has a dual effect on receptor function: first, to further enhance the receptor's kinase activity and second, to provide binding sites for signaling molecules possessing phosphotyrosine binding domains (i.e., SH2 and PTB domains) (Pawson, 2002). The complement of signaling molecules that is recruited to any given RTK dictates the cellular responses that follow receptor activation and thereby the biological activity of the pathway.

With this background, it was apparent that determining sites of receptor phosphorylation on Tie2 and the signaling molecules that associate with these 
sites would yield insights into the molecular and cellular functions of Tie2. To begin to identify signaling molecules that associate with Tie2, we constructed a recombinant Tie2 kinase domain fused to glutathione-S-transferase (GST) at the $\mathrm{N}$-terminus and to a heart muscle kinase phosphorylation site (RRASV) at the C-terminus (Huang et al., 1995). This fusion protein, termed GTEKH, could be purified efficiently on a glutathione sepharose column and ${ }^{32} \mathrm{P}$-labeled to high specific activity. Importantly, GTEKH was heavily tyrosine phosphorylated after purification, while a mutated, kinase-inactive GTEK remained unphosphorylated, strongly suggesting that GTEKH phosphorylation was due to autophosphorylation. Using radiolabeled, autophosphorylated GTEKH as an affinity reagent to probe an E. coli expression library yielded two src homology 2 (SH2) domaincontaining proteins, the adaptor protein GRB2 and the protein tyrosine phosphatase SHP2. Confirming the findings in cloning experiments, endogenous GRB2 and SHP2 from endothelial lysates associated with wild-type GTEK immobilized on glutathione agarose beads. Since phosphotyrosine residues in the C-tail of other RTKs are common sites of interaction with downstream signaling molecules, three tyrosine residues in the $\mathrm{C}$-terminal tail of Tie2 were mutated to phenylalanine (Y1101F, Y1107F, and Y1112F) (Figure 4). The Y1101F mutation markedly reduced the association of GRB2, while the SHP2 association remained intact. Conversely, the Y1112F mutation markedly decreased association of SHP2 but left GRB2 association intact. Similarly, synthetic phosphopeptides encompassing Y1101 and Y1112 specifically blocked association of GTEK with GRB2 and SHP2, respectively.

These findings indicated that GRB2 and SHP2 associate with autophosphorylated Tie2 at distinct tyrosine residues and strongly suggested a role for these signaling molecules in Tie2-mediated cellular responses and biological activity. Abundant evidence has shown that both GRB2 and SHP2 play important roles in

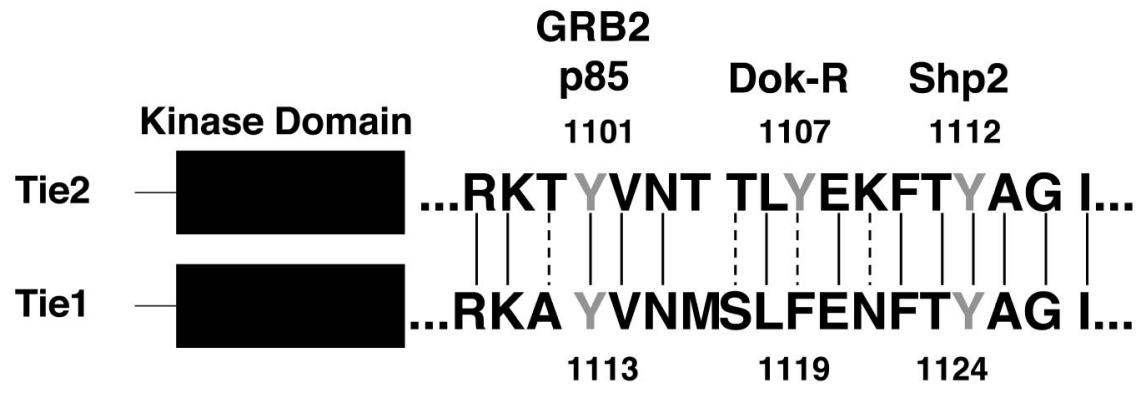

FIG. 4. Aligned peptide sequences of the C-tail tyrosine residues of Tie2 and Tie1. Some of the proteins known to associate with Tie 2 are listed above their putative sites of association. Note that tyrosine 1113 of Tie 1 conforms to the consensus sequence for binding p85. Note also that the Dok-R binding site on Tie2, Y1107, is not present on Tie1. 
the activation of the ras-mitogen-activated protein kinase (MAPK) pathway, one known to influence multiple cellular responses. Indeed, Ang1 has been shown to transiently enhance MAPK activation in cultured endothelial cells and in rat aortic ring explants, while MAPK inhibition attenuated Ang1-mediated angiogenesis in rat aortic ring explants (Fujikawa et al., 1999; Kim et al., 2002; Zhu et al., 2002). However, in cell culture, inhibition of MAPK activation had no effect on Ang1-mediated endothelial cell survival or migration (Fujikawa et al., 1999). These findings suggest that MAPK activation downstream of Tie 2 during angiogenesis may be more important for endothelial differentiation (capillary morphogenesis) than for endothelial recruitment and maintenance (Figure 5).

In addition to its positive role in MAPK activation, SHP2 may negatively regulate RTK signaling by dephosphorylating autophosphorylation sites. SHP2 phosphatase activity is enhanced by occupancy of its SH2 domains, so it is possible that recruitment of SHP2 to activated Tie2 could enhance SHP2 phosphatase activity and thereby limit phosphorylation of the activated receptor (Pluskey et al., 1995). Consistent with this possibility, mutation of the SHP2 binding site (Y1112F) on Tie2 results in enhanced autophosphorylation and

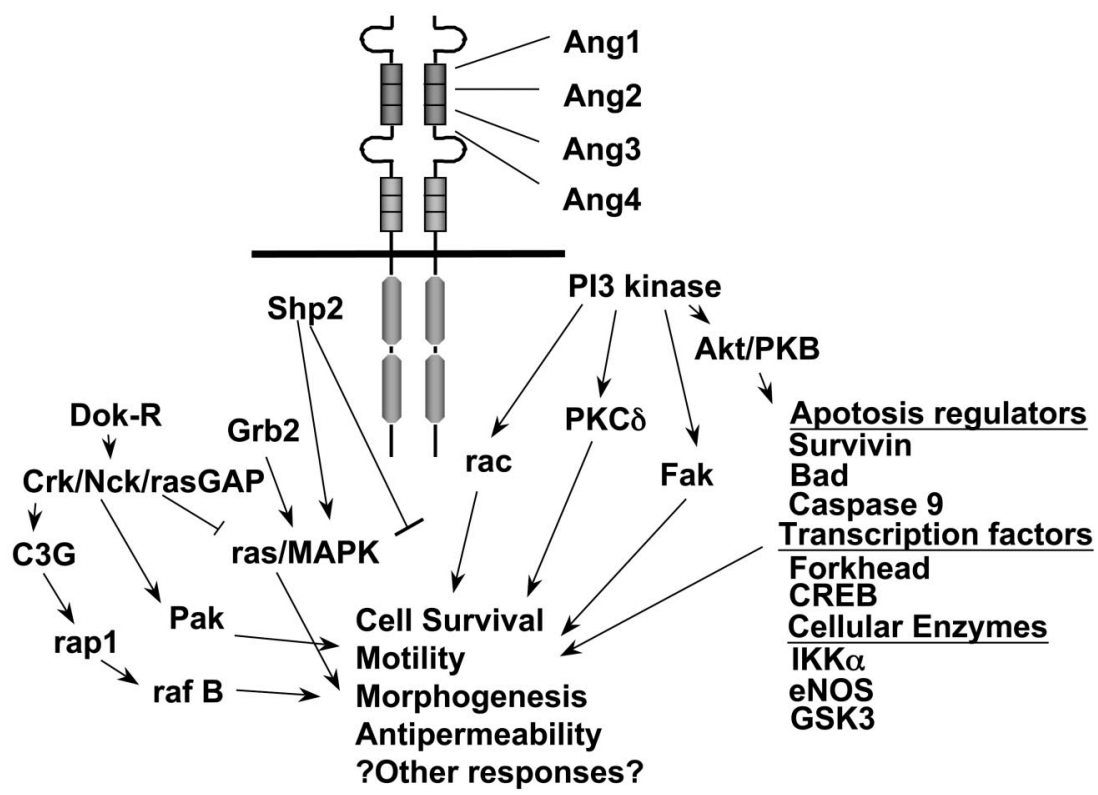

FIG. 5. Signaling pathways downstream of Tie2 activation and the cellular responses they may promote. Abbreviations: PI3, phosphatidylinositol 3; PKB, protein kinase B; PKC, protein kinase C; MAPK, mitogen-activated protein kinase; CREB, cyclic adenosine monophosphate response binding protein; IKK, I kappa B kinase complex; eNOS, endothelial nitric oxide synthase; GSK, glycogen synthase kinase. 
increased activation of downstream signaling pathways (Kontos et al., 1998; Jones et al., 2003). Recently, the structure of the Tie2 kinase domain has revealed that Y1112 is not solvent exposed but rather the phenyl ring occupies a hydrophobic pocket and the hydroxyl group forms hydrogen bonds with residues in the C-terminal lobe of the Tie2 kinase (Shewchuk et al., 2000). This positioning of Y1112 directs the C-terminal tail of Tie2 towards the substratebinding site, suggesting an autoinhibitory function. Supporting this model, a 16-residue C-tail truncation of Tie2 that includes Y1112 but spares Y1107 and Y1101 enhances the in vitro activity of the Tie2 kinase domain and yields a receptor with enhanced baseline and ligand-mediated autophosphorylation and signaling (Niu et al., 2002). Taken together, these data indicate that Y1112 may participate in two levels of negative regulation: first, to maintain Tie2 in its inactive state by obscuring the kinase active site and second, to attenuate signaling by the activated Tie 2 by recruiting SHP2. These findings increase our understanding of the molecular mechanisms of Tie2 activation and may provide insights into the development of therapeutic agents for the modulation of Tie2 activity.

Because the expression cloning approach used to identify SHP2 and GRB2 had limited ability to detect low-affinity interactions, we used a Tie2 kinase bait in the yeast two-hybrid system to screen a human fetal heart library. One of the clones identified in this screen encoded the C-terminal SH2 domain of the p85 subunit of phosphatidylinositol 3 kinase (PI3-K) (Kontos et al., 1998). Like GRB2, mutating Y1101 markedly reduced binding of p85 to Tie2 in yeastinteraction assays and in GST pull-down assays. Because Tie2 ligands were unavailable at the time, we constructed a chimeric receptor consisting of the extracellular domain of c-fms (CSF-1 receptor) and the kinase domain of Tie2 that could be activated using recombinant CSF-1. Mirroring in vitro experiments, ligand stimulation of the wild-type Tie2 kinase resulted in activation of PI3-K, as measured by accumulation of membrane-bound 3-polyinositides (predominantly PI 3,4 bisphosphate). Mutation of Y1101 to phenylalanine abrogated this effect. Further confirming the functional significance of the interaction of Tie2 with p85, CSF-1 stimulation resulted in plasma membrane translocation and activation of Akt, a serine/threonine kinase known to be a downstream effector of PI3-K. Consistent with Akt being downstream of PI3-K, CSF-1-mediated Akt activation was blocked by mutation of Y1101 and by the selective PI3-K inhibitor, wortmannin.

With discovery of the Tie2 ligands, it subsequently was shown by a number of investigators that Ang1- and, at least under certain circumstances, Ang2mediated stimulation of endogenous Tie 2 in endothelial cells resulted in the activation of Akt in a PI3-K-dependent manner (Jones et al., 1999; Kim et al., 2000a; Papapetropoulos et al., 2000). Moreover, these studies demonstrated that activation of the PI3-K/Akt pathway was required for Ang1-mediated cell 
survival and chemotaxis. As mentioned previously, these responses were not inhibited by a selective MAPK inhibitor (Fujikawa et al., 1999). A more-recent study has shown that the adaptor protein, Dok-R, is recruited to activated Tie 2 by association of its protein tyrosine binding (PTB) domain with Y1107 (Jones et al., 2003). Dok-R subsequently is phosphorylated on multiple tyrosine residues, resulting in recruitment of additional signaling molecules with putative roles in a number of cellular responses, including cell migration. Predictably, recruitment and phosphorylation of Dok-R were attenuated by mutation of Y1107, as was the chemotactic response to Ang1. Surprisingly, however, association and phosphorylation of Dok-R also were attenuated by mutation of Y1101, by mutation of the Dok-R PH domain, or by treatment of cells with a PI3-K inhibitor. These findings suggested that the signaling cross-talk between the PI3-K pathway and the Dok-R pathway is required for an optimal chemotactic response downstream of Tie2. Other recent studies have shown that Tie2-mediated activation of the PI3-K/Akt pathway is required for endothelial nitric oxide synthase (eNOS) activation, focal adhesion kinase activation, and protease secretion, all of which may contribute importantly to Tie2 function during angiogenesis (Kim et al., 2000c; Babaei et al., 2003). Supporting this idea, eNOS KO mice had a markedly reduced angiogenic response to Ang1 in the Matrigel plug assay (Babaei et al., 2003). Based on these studies, it is likely that PI3-K activation and PI3-Kdependent cellular responses are pivotal for the function of Tie2 in the maintenance and in the angiogenic response of the adult vasculature.

Like Tie2, the closely related Tie1 receptor is required for normal embryonic vascular development. We recently have shown that activation of a c-fms/Tie1 receptor also stimulates the PI3-K/Akt survival pathway, suggesting a similar role for Tie1 in maintenance of the adult vasculature (Kontos et al., 2002). Consistent with this possibility, in chimeric WT/Tie1 KO mice, Tie1 null endothelial cells contribute normally to the vasculature until E10.5 but are increasingly underrepresented after E12.5 and into adulthood (Puri et al., 1995). These studies indicate that the Tie1 and Tie2 pathways have both overlapping and nonoverlapping functions. Although a ligand for Tie1 has yet to be identified, our study using a cfms/Tie1 chimeric receptor suggests that Tie1 may be activated by ligand-mediated dimerization. However, another recent study suggests that Tie1 may be activated via a heterotypic interaction with Tie2 (Marron et al., 2000). Clarification of the mechanism by which Tie1 is activated will be important to fully understand the relationship and function of these two crucial endothelial signaling pathways.

\section{Therapeutic Modulation of Tie2 Activation}

The studies described above implicate Tie 2 signaling in vascular maintenance and in angiogenesis in adult tissues and suggest that therapeutic modula- 
tion of Tie2 activation may be beneficial. However, important questions remain unanswered. Perhaps the most-pressing unanswered question is the mechanism underlying the differential effects of Ang1 and Ang2 on Tie2 activation. Recent data have demonstrated that the C-terminal fibrinogen domain is required for Tie2 binding of both ligands, suggesting that differential oligomerization of Ang1 vs. Ang2 might be responsible for the observed functional differences (Procopio et al., 1999; Davis et al., 2003). However, in endothelial cells (ECs), dimers of either Ang1 or Ang2 fail to activate Tie2, whereas tetramers of Ang1 activate Tie2 but tetramers of Ang2 not only fail to activate Tie2 but inhibit binding of Ang1 tetramers (Davis et al., 2003). Based on these findings, it was suggested that a subtle difference in the binding of Ang1 vs, Ang2 fibrinogen domains might be responsible for their contrasting activities on ECs. However, another group has shown that the binding sites for Ang1 and Ang2 on Tie2 are grossly indistinguishable (Fiedler et al., 2003). In view of these data, it may be more likely that differences between Ang1 and Ang2 are due to expression of an Ang2 coreceptor in endothelial cells. The expression of such a coreceptor, or lack thereof, might explain the different responses of lymphatic vs. blood vascular endothelium and perhaps some of the apparently disparate effects of Ang1 and ExTek on pathological angiogenesis.

Based a broad range of data, we must continue to explore the therapeutic potential of modulating the activity of the Tie 2 pathway. From our studies and those of many others, it appears that therapeutic manipulation of angiogenesis will likely be an important clinical application of Tie2 biology. Most of the data suggest that activation of Tie 2 plays an important role in blood vessel assembly and that inhibiting the Tie2 pathway will inhibit pathological angiogenesis. Although the therapeutic utility of soluble receptors has been shown for other ligand/receptor systems, further proof of concept of Tie2 inhibition for antiangiogenesis would be greatly facilitated by the advent of potent and selective small molecule inhibitors of the Tie2 kinase. Such inhibitors would be powerful tools to explore the role of Tie2 in quiescent adult vasculature.

In addition to antiangiogenesis, recent data suggest that enhancing Tie2 activation can enhance angiogenesis in normal tissues and improve collateral development and blood flow to ischemic tissues. For example, transgenic overexpression of Ang1 in the skin increased dermal vascularity and exogenous delivery of Ang1 alone or in combination with VEGF improved collateral development and perfusion to ischemic skeletal muscle (Shyu et al., 1998; Suri et al., 1998; Chae et al., 2000). In addition to its pro-angiogenic effects, Tie2 activation attenuates the action of pro-inflammatory mediators and decreases vascular leak, suggesting that Tie 2 activation may have other applications in vascular disease, such as plaque stabilization in atherosclerosis and limiting cerebral edema after ischemic stroke (Thurston et al., 1999; Gamble et al., 2000; Kim et al., 2001). Indeed, recent studies have shown that Ang1 is protective in 
rodent models of pulmonary hypertension and allograft atherosclerosis (Nykanen et al., 2003; Zhao et al., 2003). Additionally, since VEGF enhances expression of inflammatory mediators and increases vascular permeability, coadministration of Ang1 with VEGF could represent an improved approach to proangiogenesis. Continued improvement of gene transfer vectors for delivery of endogenous Tie2 ligands or continued development of recombinant Tie2 ligands that are more homogeneous and easier to produce could yield effective therapeutic agents for ischemic and other vascular disorders. Alternative strategies to enhance Tie2 activation - such as direct kinase activators or inhibition of protein tyrosine phosphatases such as SHP2 and others that negatively regulate Tie 2 - may be effective future therapeutic tools.

Although a tempting therapeutic target, modulation of Tie 2 signaling in the adult vasculature should be approached with caution. Data presented earlier indicate that Tie2 likely plays a role in maintenance of the adult vasculature, perhaps mediating endothelial survival. Our studies with ExTek, however, suggest that at least short-term inhibition of Tie2 signaling will be well tolerated. Improved approaches for conditional KOs of Tie 2 or the development of potent and selective Tie2 inhibitors will be necessary to determine the effects of chronic blockade of the Tie2 pathway in adult vasculature. It is possible that enhanced Tie 2 signaling may have adverse consequences in the adult vasculature. We and others have shown that mutations in Tie2 that cause inherited venous malformations enhance ligandindependent and ligand-dependent Tie2 kinase activity (Vikkula et al., 1996; Calvert et al., 1999). Despite these findings, chronic expression of Ang1 in murine skin enhanced dermal vascularity without apparent adverse effects. In addition, studies in animal models of ischemia indicate that short-term enhancement of Tie 2 signaling is sufficient to improve collateral circulation without evidence of untoward effects. Taken together, these data strongly suggest that, although caution is warranted, therapeutic modulation of the Tie2 pathway to inhibit or enhance blood vessel growth and remodeling will have a favorable therapeutic index.

\section{ACKNOWLEDGMENT}

We gratefully acknowledge the administrative support of Katie Hunter, whose enthusiasm and untiring efforts contributed importantly to the success of our studies.

\section{REFERENCES}

Babaei S, Teichert-Kuliszewska K, Zhang Q, Jones N, Dumont DJ, Stewart DJ 2003 Angiogenic actions of angiopoietin-1 require endothelium-derived nitric oxide. Am J Pathol 162:19271936 
Calvert JT, Riney TJ, Kontos CD, Cha EH, Prieto VG, Shea CR, Berg JN, Nevin NC, Simpson SA, Pasyk KA, Speer MC, Peters KG, Marchuk DA 1999 Allelic and locus heterogeneity in inherited venous malformations. Hum Mol Genet 8:1279-1289

Chae JK, Kim I, Lim ST, Chung MJ, Kim WH, Kim HG, Ko JK, Koh GY 2000 Coadministration of angiopoietin-1 and vascular endothelial growth factor enhances collateral vascularization. Arterioscler Thromb Vasc Biol 20:2573-2578

Conway EM, Collen D, Carmeliet P 2001 Molecular mechanisms of blood vessel growth. Cardiovasc Res 49:507-521

Davis S, Aldrich TH, Jones PF, Acheson A, Compton DL, Jain V, Ryan TE, Bruno J, Radziejewski C, Maisonpierre PC, Yancopoulos GD 1996 Isolation of angiopoietin-1, a ligand for the TIE2 receptor, by secretion-trap expression cloning. Cell 87:1161-1169

Davis S, Papadopoulos N, Aldrich TH, Maisonpierre PC, Huang T, Kovac L, Xu A, Leidich R, Radziejewska E, Rafique A, Goldberg J, Jain V, Bailey K, Karow M, Fandl J, Samuelsson SJ, Ioffe E, Rudge JS, Daly TJ, Radziejewski C, Yancopoulos GD 2003 Angiopoietins have distinct modular domains essential for receptor binding, dimerization and superclustering. Nature Struct Biol 10:38-44

Dumont DJ, Yamaguchi TP, Conlon RA, Rossant J, Breitman ML 1992 tek, a novel tyrosine kinase gene located on mouse chromosome 4, is expressed in endothelial cells and their presumptive precursors. Oncogene 7:1471-1480

Dumont DJ, Gradwohl G, Fong GH, Puri MC, Gertsenstein M, Auerbach A, Breitman ML 1994 Dominant-negative and targeted null mutations in the endothelial receptor tyrosine kinase, tek, reveal a critical role in vasculogenesis of the embryo. Genes Dev 8:1897-1909

Fiedler U, Krissl T, Koidl S, Weiss C, Koblizek T, Deutsch U, Martiny-Baron G, Marme D, Augustin HG 2003 Angiopoietin-1 and angiopoietin-2 share the same binding domains in the Tie-2 receptor involving the first Ig-like loop and the epidermal growth factor-like repeats. J Biol Chem 278:1721-1727

Folkman J 2001 Angiogenesis-dependent diseases. Semin Oncol 28:536-542

Forde A, Constien R, Grone HJ, Hammerling G, Arnold B 2002 Temporal Cre-mediated recombination exclusively in endothelial cells using Tie2 regulatory elements. Genesis 33:191-197

Fujikawa K, de AS, I, Jain SK, Presman E, Christensen RA, Varticovski L 1999 Role of PI 3-kinase in angiopoietin-1-mediated migration and attachment-dependent survival of endothelial cells. Exp Cell Res 253:663-672

Gale NW, Thurston G, Hackett SF, Renard R, Wang Q, McClain J, Martin C, Witte C, Witte MH, Jackson D, Suri C, Campochiaro PA, Wiegand SJ, Yancopoulos GD 2002 Angiopoietin-2 is required for postnatal angiogenesis and lymphatic patterning, and only the latter role is rescued by Angiopoietin-1. Dev Cell 3:411-423

Gamble JR, Drew J, Trezise L, Underwood A, Parsons M, Kasminkas L, Rudge J, Yancopoulos G, Vadas MA 2000 Angiopoietin-1 is an antipermeability and anti-inflammatory agent in vitro and targets cell junctions. Circ Res 87:603-607

Hackett SF, Ozaki H, Strauss RW, Wahlin K, Suri C, Maisonpierre P, Yancopoulos G, Campochiaro PA 2000 Angiopoietin 2 expression in the retina: upregulation during physiologic and pathologic neovascularization. J Cell Physiol 184:275-284

Hangai M, Moon YS, Kitaya N, Chan CK, Wu DY, Peters KG, Ryan SJ, Hinton DR 2001 Systemically expressed soluble Tie2 inhibits intraocular neovascularization. Hum Gene Ther 12:1311-1321

Hata K, Udagawa J, Fujiwaki R, Nakayama K, Otani H, Miyazaki K 2002 Expression of angiopoietin-1, angiopoietin-2, and Tie2 genes in normal ovary with corpus luteum and in ovarian cancer. Oncology 62:340-348 
Hawighorst T, Skobe M, Streit M, Hong YK, Velasco P, Brown LF, Riccardi L, LangeAsschenfeldt B, Detmar M 2002 Activation of the tie2 receptor by angiopoietin-1 enhances tumor vessel maturation and impairs squamous cell carcinoma growth. Am J Pathol 160: $1381-1392$

Hayes AJ, Huang WQ, Yu J, Maisonpierre PC, Liu A, Kern FG, Lippman ME, McLeskey SW, Li LY 2000 Expression and function of angiopoietin-1 in breast cancer. Br J Cancer 83:1154-1160

Holash J, Maisonpierre PC, Compton D, Boland P, Alexander CR, Zagzag D, Yancopoulos GD, Wiegand SJ 1999 Vessel cooption, regression, and growth in tumors mediated by angiopoietins and VEGF. Science 284:1994-1998

Huang L, Turck CW, Rao P, Peters KG 1995 GRB2 and SH-PTP2: potentially important endothelial signaling molecules downstream of the TEK/TIE2 receptor tyrosine kinase. Oncogene 11:2097-2103

Iwama A, Hamaguchi I, Hashiyama M, Murayama Y, Yasunaga K, Suda T 1993 Molecular cloning and characterization of mouse TIE and TEK receptor tyrosine kinase genes and their expression in hematopoietic stem cells. Biochem Biophys Res Commun 195:301-309

Jones N, Master Z, Jones J, Bouchard D, Gunji Y, Sasaki H, Daly R, Alitalo K, Dumont DJ 1999 Identification of Tek/Tie2 binding partners. Binding to a multifunctional docking site mediates cell survival and migration. J Biol Chem 274:30896-30905

Jones N, Iljin K, Dumont DJ, Alitalo K 2001a Tie receptors: new modulators of angiogenic and lymphangiogenic responses. Nature Rev Mol Cell Biol 2:257-267

Jones N, Voskas D, Master Z, Sarao R, Jones J, Dumont DJ 2001b Rescue of the early vascular defects in Tek/Tie2 null mice reveals an essential survival function. EMBO Rep 2:438-445

Jones N, Chen SH, Sturk C, Master Z, Tran J, Kerbel RS, Dumont DJ 2003 A unique autophosphorylation site on Tie2/Tek mediates Dok-R phosphotyrosine binding domain binding and function. Mol Cell Biol 23:2658-2668

Kim I, Kim JH, Moon SO, Kwak HJ, Kim NG, Koh GY 2000a Angiopoietin-2 at high concentration can enhance endothelial cell survival through the phosphatidylinositol $3^{\prime}$ kinase/Akt signal transduction pathway. Oncogene 19:4549-4552

Kim I, Kim HG, So JN, Kim JH, Kwak HJ, Koh GY 2000b Angiopoietin-1 regulates endothelial cell survival through the phosphatidylinositol 3'-Kinase/Akt signal transduction pathway. Circ Res 86:24-29

Kim I, Kim HG, Moon SO, Chae SW, So JN, Koh KN, Ahn BC, Koh GY 2000c Angiopoietin-1 induces endothelial cell sprouting through the activation of focal adhesion kinase and plasmin secretion. Circ Res 86:952-959

Kim I, Moon SO, Park SK, Chae SW, Koh GY 2001 Angiopoietin-1 reduces VEGF-stimulated leukocyte adhesion to endothelial cells by reducing ICAM-1, VCAM-1, and E-selectin expression. Circ Res 89:477-479

Kim I, Ryu YS, Kwak HJ, Ahn SY, Oh JL, Yancopoulos GD, Gale NW, Koh GY 2002 EphB ligand, ephrinB2, suppresses the VEGF- and angiopoietin 1-induced Ras/mitogen-activated protein kinase pathway in venous endothelial cells. FASEB J 16:1126-1128

Kisanuki YY, Hammer RE, Miyazaki J, Williams SC, Richardson JA, Yanagisawa M 2001 Tie2-Cre transgenic mice: a new model for endothelial cell-lineage analysis in vivo. Dev Biol 230:230-242

Koga K, Todaka T, Morioka M, Hamada J, Kai Y, Yano S, Okamura A, Takakura N, Suda T, Ushio Y 2001 Expression of angiopoietin-2 in human glioma cells and its role for angiogenesis. Cancer Res 61:6248-6254

Kontos CD, Stauffer TP, Yang WP, York JD, Huang L, Blanar MA, Meyer T, Peters KG 1998 Tyrosine 1101 of Tie2 is the major site of association of p 85 and is required for activation of phosphatidylinositol 3-kinase and Akt. Mol Cell Biol 18:4131-4140 
Kontos CD, Cha EH, York JD, Peters KG 2002 The endothelial receptor tyrosine kinase Tie1 activates phosphatidylinositol 3-kinase and Akt to inhibit apoptosis. Mol Cell Biol 22:17041713

Lin P, Polverini P, Dewhirst M, Shan S, Rao PS, Peters K 1997 Inhibition of tumor angiogenesis using a soluble receptor establishes a role for Tie2 in pathologic vascular growth. J Clin Invest 100:2072-2078

Lin P, Buxton JA, Acheson A, Radziejewski C, Maisonpierre PC, Yancopoulos GD, Channon KM, Hale LP, Dewhirst MW, George SE, Peters KG 1998a Antiangiogenic gene therapy targeting the endothelium-specific receptor tyrosine kinase Tie2. Proc Natl Acad Sci USA 95:8829-8834

Lin P, Sankar S, Shan S, Dewhirst MW, Polverini PJ, Quinn TQ, Peters KG 1998b Inhibition of tumor growth by targeting tumor endothelium using a soluble vascular endothelial growth factor receptor. Cell Growth Differ 9:49-58

Loughna S, Sato TN 2001 Angiopoietin and Tie signaling pathways in vascular development. Matrix Biol 20:319-325

Lyons MS, Bell B, Stainier D, Peters KG 1998 Isolation of the zebrafish homologues for the tie-1 and tie-2 endothelium-specific receptor tyrosine kinases. Dev Dyn 212:133-140

Maisonpierre PC, Goldfarb M, Yancopoulos GD, Gao G 1993 Distinct rat genes with related profiles of expression define a TIE receptor tyrosine kinase family. Oncogene 8:1631-1637

Maisonpierre PC, Suri C, Jones PF, Bartunkova S, Wiegand SJ, Radziejewski C, Compton D, McClain J, Aldrich TH, Papadopoulos N, Daly TJ, Davis S, Sato TN, Yancopoulos GD 1997 Angiopoietin-2, a natural antagonist for Tie2 that disrupts in vivo angiogenesis. Science 277:55-60

Marron MB, Hughes DP, Edge MD, Forder CL, Brindle NP 2000 Evidence for heterotypic interaction between the receptor tyrosine kinases TIE-1 and TIE-2. J Biol Chem 275:3974139746

Motoike T, Loughna S, Perens E, Roman BL, Liao W, Chau TC, Richardson CD, Kawate T, Kuno J, Weinstein BM, Stainier DY, Sato TN 2000 Universal GFP reporter for the study of vascular development. Genesis 28:75-81

Niu XL, Peters KG, Kontos CD 2002 Deletion of the carboxyl terminus of Tie2 enhances kinase activity, signaling, and function. Evidence for an autoinhibitory mechanism. J Biol Chem 277:31768-31773

Nykanen AI, Krebs R, Saaristo A, Turunen P, Alitalo K, Yla-Herttuala S, Koskinen PK, Lemstrom KB 2003 Angiopoietin-1 protects against the development of cardiac allograft arteriosclerosis. Circulation 107:1308-1314

Papapetropoulos A, Fulton D, Mahboubi K, Kalb RG, O'Connor DS, Li F, Altieri DC, Sessa WC 2000 Angiopoietin-1 inhibits endothelial cell apoptosis via the Akt/survivin pathway. J Biol Chem 275:9102-9105

Pawson T 2002 Regulation and targets of receptor tyrosine kinases. Eur J Cancer 38(suppl 5):S3-S10

Peters KG, Coogan A, Berry D, Marks J, Iglehart JD, Kontos CD, Rao P, Sankar S, Trogan E 1998 Expression of Tie2/Tek in breast tumour vasculature provides a new marker for evaluation of tumour angiogenesis. Br J Cancer 77:51-56

Pluskey S, Wandless TJ, Walsh CT, Shoelson SE 1995 Potent stimulation of SH-PTP2 phosphatase activity by simultaneous occupancy of both SH2 domains. J Biol Chem 270:2897-2900

Procopio WN, Pelavin PI, Lee WM, Yeilding NM 1999 Angiopoietin-1 and -2 coiled coil domains mediate distinct homo-oligomerization patterns, but fibrinogen-like domains mediate ligand activity. J Biol Chem 274:30196-30201

Puri MC, Rossant J, Alitalo K, Bernstein A, Partanen J 1995 The receptor tyrosine kinase TIE is required for integrity and survival of vascular endothelial cells. EMBO J 14:5884-5891 
Puri MC, Partanen J, Rossant J, Bernstein A 1999 Interaction of the TEK and TIE receptor tyrosine kinases during cardiovascular development. Development 126:4569-4580

Runting AS, Stacker SA, Wilks AF 1993 tie2, a putative protein tyrosine kinase from a new class of cell surface receptor. Growth Factors 9:99-105

Sato TN, Qin Y, Kozak CA, Audus KL 1993 Tie-1 and tie-2 define another class of putative receptor tyrosine kinase genes expressed in early embryonic vascular system. Proc Natl Acad Sci USA 90:9355-9358

Sato TN, Tozawa Y, Deutsch U, Wolburg-Buchholz K, Fujiwara Y, Gendron-Maguire M, Gridley T, Wolburg H, Risau W, Qin Y 1995 Distinct roles of the receptor tyrosine kinases Tie-1 and Tie-2 in blood vessel formation. Nature 376:70-74

Schlaeger TM, Bartunkova S, Lawitts JA, Teichmann G, Risau W, Deutsch U, Sato TN 1997 Uniform vascular-endothelial-cell-specific gene expression in both embryonic and adult transgenic mice. Proc Natl Acad Sci USA 94:3058-3063

Schlessinger J 2000 Cell signaling by receptor tyrosine kinases. Cell 103:211-225

Schnurch H, Risau W 1993 Expression of tie-2, a member of a novel family of receptor tyrosine kinases, in the endothelial cell lineage. Development 119:957-968

Shewchuk LM, Hassell AM, Ellis B, Holmes WD, Davis R, Horne EL, Kadwell SH, McKee DD, Moore JT 2000 Structure of the Tie2 RTK domain: self-inhibition by the nucleotide binding loop, activation loop, and C-terminal tail. Structure Fold Des 8:1105-1113

Shim WS, Teh M, Mack PO, Ge R 2001 Inhibition of angiopoietin-1 expression in tumor cells by an antisense RNA approach inhibited xenograft tumor growth in immunodeficient mice. Int J Cancer 94:6-15

Shim WS, Teh M, Bapna A, Kim I, Koh GY, Mack PO, Ge R 2002 Angiopoietin 1 promotes tumor angiogenesis and tumor vessel plasticity of human cervical cancer in mice. Exp Cell Res 279:299-309

Shyu KG, Manor O, Magner M, Yancopoulos GD, Isner JM 1998 Direct intramuscular injection of plasmid DNA encoding angiopoietin-1 but not angiopoietin-2 augments revascularization in the rabbit ischemic hindlimb. Circulation 98:2081-2087

Siemeister G, Schirner M, Weindel K, Reusch P, Menrad A, Marme D, Martiny-Baron G 1999 Two independent mechanisms essential for tumor angiogenesis: inhibition of human melanoma xenograft growth by interfering with either the vascular endothelial growth factor receptor pathway or the Tie-2 pathway. Cancer Res 59:3185-3191

Stoeltzing O, Ahmad SA, Liu W, McCarty MF, Parikh AA, Fan F, Reinmuth N, Bucana CD, Ellis LM 2002 Angiopoietin-1 inhibits tumour growth and ascites formation in a murine model of peritoneal carcinomatosis. Br J Cancer 87:1182-1187

Stoeltzing O, Ahmad SA, Liu W, McCarty MF, Wey JS, Parikh AA, Fan F, Reinmuth N, Kawaguchi M, Bucana CD, Ellis LM 2003 Angiopoietin-1 inhibits vascular permeability, angiogenesis, and growth of hepatic colon cancer tumors. Cancer Res 63:3370-3377

Stratmann A, Risau W, Plate KH 1998 Cell type-specific expression of angiopoietin-1 and angiopoietin-2 suggests a role in glioblastoma angiogenesis. Am J Pathol 153:1459-1466

Stratmann A, Acker T, Burger AM, Amann K, Risau W, Plate KH 2001 Differential inhibition of tumor angiogenesis by tie2 and vascular endothelial growth factor receptor-2 dominantnegative receptor mutants. Intl J Cancer 91:273-282

Suri C, Jones PF, Patan S, Bartunkova S, Maisonpierre PC, Davis S, Sato TN, Yancopoulos GD 1996 Requisite role of angiopoietin-1, a ligand for the TIE2 receptor, during embryonic angiogenesis. Cell 87:1171-1180

Suri C, McClain J, Thurston G, McDonald DM, Zhou H, Oldmixon EH, Sato TN, Yancopoulos GD 1998 Increased vascularization in mice overexpressing angiopoietin-1. Science 282:468471 
Takagi H, Koyama S, Seike H, Oh H, Otani A, Matsumura M, Honda Y 2003 Potential role of the angiopoietin/tie2 system in ischemia-induced retinal neovascularization. Invest Ophthalmol Vis Sci 44:393-402

Takahashi T, Takahashi K, St John PL, Fleming PA, Tomemori T, Watanabe T, Abrahamson DR, Drake CJ, Shirasawa T, Daniel TO 2003 A mutant receptor tyrosine phosphatase, CD148, causes defects in vascular development. Mol Cell Biol 23:1817-1831

Tanaka S, Mori M, Sakamoto Y, Makuuchi M, Sugimachi K, Wands JR 1999 Biologic significance of angiopoietin-2 expression in human hepatocellular carcinoma. J Clin Invest 103:341-345

Tanaka S, Sugimachi K, Yamashita YY, Ohga T, Shirabe K, Shimada M, Wands JR, Sugimachi K 2002 Tie2 vascular endothelial receptor expression and function in hepatocellular carcinoma. Hepatology 35:861-867

Teichert-Kuliszewska K, Maisonpierre PC, Jones N, Campbell AI, Master Z, Bendeck MP, Alitalo K, Dumont DJ, Yancopoulos GD, Stewart DJ 2001 Biological action of angiopoietin-2 in a fibrin matrix model of angiogenesis is associated with activation of Tie2. Cardiovasc Res 49:659-670

Theis M, de Wit C, Schlaeger TM, Eckardt D, Kruger O, Doring B, Risau W, Deutsch U, Pohl U, Willecke K 2001 Endothelium-specific replacement of the connexin43 coding region by a lacZ reporter gene. Genesis 29:1-13

Thurston G, Suri C, Smith K, McClain J, Sato TN, Yancopoulos GD, McDonald DM 1999 Leakage-resistant blood vessels in mice transgenically overexpressing angiopoietin-1. Science 286:2511-2514

Valenzuela DM, Griffiths JA, Rojas J, Aldrich TH, Jones PF, Zhou H, McClain J, Copeland NG, Gilbert DJ, Jenkins NA, Huang T, Papadopoulos N, Maisonpierre PC, Davis S, Yancopoulos GD 1999 Angiopoietins 3 and 4: diverging gene counterparts in mice and humans. Proc Natl Acad Sci USA 96:1904-1909

Valtola R, Salven P, Heikkila P, Taipale J, Joensuu H, Rehn M, Pihlajaniemi T, Weich H, deWaal R, Alitalo K 1999 VEGFR-3 and its ligand VEGF-C are associated with angiogenesis in breast cancer. Am J Pathol 154:1381-1390

Vicent D, Ilany J, Kondo T, Naruse K, Fisher SJ, Kisanuki YY, Bursell S, Yanagisawa M, King GL, Kahn CR 2003 The role of endothelial insulin signaling in the regulation of vascular tone and insulin resistance. J Clin Invest 111:1373-1380

Vikkula M, Boon LM, Carraway KL III, Calvert JT, Diamonti AJ, Goumnerov B, Pasyk KA, Marchuk DA, Warman ML, Cantley LC, Mulliken JB, Olsen BR 1996 Vascular dysmorphogenesis caused by an activating mutation in the receptor tyrosine kinase TIE2. Cell 87:1181-1190

Witzenbichler B, Maisonpierre PC, Jones P, Yancopoulos GD, Isner JM 1998 Chemotactic properties of angiopoietin-1 and -2 , ligands for the endothelial-specific receptor tyrosine kinase Tie2. J Biol Chem 273:18514-18521

Wong AL, Haroon ZA, Werner S, Dewhirst MW, Greenberg CS, Peters KG 1997 Tie2 expression and phosphorylation in angiogenic and quiescent adult tissues. Circ Res 81:567574

Yancopoulos GD, Davis S, Gale NW, Rudge JS, Wiegand SJ, Holash J 2000 Vascular-specific growth factors and blood vessel formation. Nature 407:242-248

Zagzag D, Hooper A, Friedlander DR, Chan W, Holash J, Wiegand SJ, Yancopoulos GD, Grumet M 1999 In situ expression of angiopoietins in astrocytomas identifies angiopoietin-2 as an early marker of tumor angiogenesis. Exp Neurol 159:391-400

Zhao YD, Campbell AI, Robb M, Ng D, Stewart DJ 2003 Protective role of angiopoietin-1 in experimental pulmonary hypertension. Circ Res 92:984-991 
Zhu WH, MacIntyre A, Nicosia RF 2002 Regulation of angiogenesis by vascular endothelial growth factor and angiopoietin-1 in the rat aorta model: distinct temporal patterns of intracellular signaling correlate with induction of angiogenic sprouting. Am J Pathol 161: $823-830$

Ziegler SF, Bird TA, Schneringer JA, Schooley KA, Baum PR 1993 Molecular cloning and characterization of a novel receptor protein tyrosine kinase from human placenta. Oncogene 8:663-670 